\title{
High accuracy Raman measurements using the Stokes and anti-Stokes lines
}

\author{
Witold Trzeciakowski, ${ }^{\text {a) }}$ Juan Martínez-Pastor, and Andrés Cantarero \\ Departamento de Física Aplicado, Universidad de Valencia, 46100 Burjasot Valencia, Spain
}

(Received 23 April 1997; accepted for publication 5 July 1997)

\begin{abstract}
We show that by measuring the separation between the Stokes and anti-Stokes peaks excited by two different laser lines we obtain a very precise determination of absolute phonon energies. The method is useful for measuring small changes of these energies with strain, temperature, laser power, etc. It doubles the changes and avoids the necessity of using the reference lines in the Raman spectra. The method can be applied for the determination of phonon deformation potentials, for the characterization of strained heteroepitaxial layers, and for micro-Raman analysis of strain in silicon integrated circuits. We give examples of phonon shifts in $\mathrm{Si}, \mathrm{Ge}, \mathrm{GaAs}$, InAs, and $\mathrm{GaP}$ as a function of applied biaxial strain, laser power, and temperature. (c) 1997 American Institute of Physics. [S0021-8979(97)02120-8]
\end{abstract}

\section{INTRODUCTION}

Te literature values of optical-phonon frequencies in various semiconductors show a scatter of a few wave numbers. Even under the same conditions of temperature, strain, energy density of the laser spot, etc. the position of the peaks in Raman measurements performed on the same sample may show a scatter of up to $1 \mathrm{~cm}^{-1}$. This may be due to small errors in the steps of the monochromator, to errors in the calibration of the zero, etc. In many cases, high accuracy of the absolute energies of the phonons is not necessary but there are important examples of the contrary. For instance, Raman spectroscopy has been used as a tool for the characterization of strained-layer heterostructures ${ }^{1-4}$ or for the determination of strain in silicon integrated circuits. ${ }^{5,6}$ In these cases it is important to know the values of the phonon frequencies in the unstrained case and to determine their small variations. Also, determination of the phonon deformation potentials from uniaxial measurements requires an accuracy of about $0.1 \mathrm{~cm}^{-1}$. Similar accuracy is desirable for measuring the phonon shifts with temperature (around $2 \mathrm{~cm}^{-1}$ per $100 \mathrm{~K}$ ) or with laser power. The solution to that problem may be a reference line in the spectra. This may be the sharp line from a spectral lamp ${ }^{7}$ or a plasma line from the laser. ${ }^{5}$ The plasma lines seem more convenient because they arrive in the same beam as the exciting line. However, it is not always possible to find a plasma line in the appropriate spectral range around an arbitrary laser line. In this article we propose another method which involves two laser lines and allows one to determine absolute phonon energies (and their shifts) with $0.1 \mathrm{~cm}^{-1}$ accuracy. The method doubles the shift of the lines, which is important when we consider small effects of the order of $1 \mathrm{~cm}^{-1}$ (the typical range in uniaxial stress experiments ${ }^{7,8}$ ). It works well at room temperature (and above) for all materials that have a decent Raman signal so the anti-Stokes line can be measured. It is not applicable at low temperatures when the anti-Stokes lines disappear.

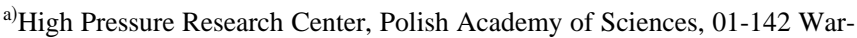
saw, Poland.
}

\section{THE METHOD AND ITS EXPERIMENTAL IMPLEMENTATION}

Let us imagine that the sample is illuminated simultaneously with two laser lines ( $E_{1}^{L}$ and $E_{2}^{L}$ in Fig. 1). Each of these lines gives rise to the Stokes and anti-Stokes peaks (here we assume that there is only one phonon energy) denoted by ES and EAS, respectively. The relative intensities $I^{S} / I^{A S}$ for a given laser line should be approximately equal to $\exp \left(\hbar \omega_{0} / k T\right)$, where $\hbar \omega_{0}$ is the phonon energy. At room temperature, $k T \approx 24 \mathrm{meV}$ and the optical-phonon energies lie in the range $25-65 \mathrm{meV}$ so that the Stokes line can be much stronger than the anti-Stokes one (see Table I). If the separation of the laser lines is not far from two times the phonon energy $2 \hbar \omega$, then the Stokes and anti-Stokes lines $E_{2}^{S}$ and $E_{1}^{A S}$ will be close to each other and their separation can be measured to a high accuracy (say, $0.1 \mathrm{~cm}^{-1}$ ). As the separation of the laser lines is known to a higher accuracy, we can determine the phonon energy from the simple equation:

$$
\hbar \omega_{0}=\frac{1}{2}\left[\left(E_{2}^{L}-E_{1}^{L}\right)-\left(E_{2}^{S}-E_{1}^{A S}\right)\right] .
$$

This equation determines absolute phonon energies with $0.05 \mathrm{~cm}^{-1}$ accuracy. Small changes of $\hbar \omega_{0}$ (due to strain, temperature, etc.) can then be detected with high precision because $E_{2}^{S}$ and $E_{1}^{A S}$ will be shifted in the opposite directions (i.e., the changes are multiplied by a factor of 2). The separation of the laser lines can be either greater or smaller than $2 \hbar \omega_{0}$; in the latter case the order of the Stokes and antiStokes peaks would be reversed.

An obvious question arises: Can we find appropriate pairs of laser lines such that their separation is not far from $2 \hbar \omega_{0}$ for a given material? In Table I we list the possible pairs of lines of an argon laser that can be used for the determination of phonon energies in different semiconductors. We considered the following wavelengths (in $\mathrm{nm}$ ): 528.7, 514.5, 501.7, 496.5, 488.0, 476.5, 472.7, 465.8, 457.9, $454.5,351.1$, and 363.8 , which are available in some models. We assumed that we are interested in the Raman spectrum between $\hbar \omega_{\text {LO }}$ and $\hbar \omega_{\text {TO }}$ [longitudinal optical (LO) and transverse optical (TO)]. Therefore we looked for the pairs of 


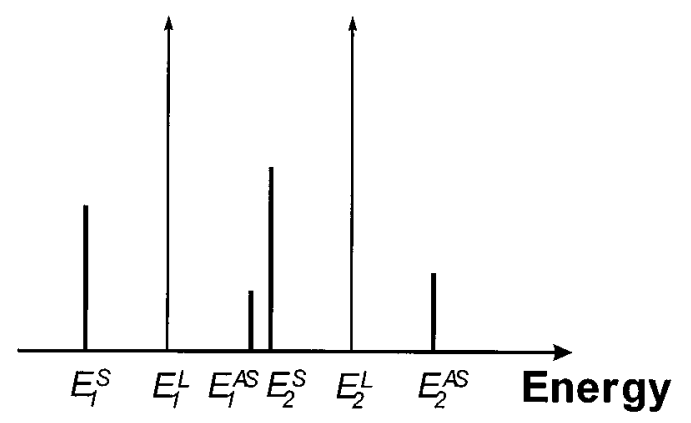

FIG. 1. Schematic idea of the Stokes/anti-Stokes method. Two laser lines $E_{1}^{L}$ and $E_{2}^{L}$ lead to Stokes $E^{S}$ and anti-Stokes $E^{A S}$ peaks around each line. By measuring the (small) separation between $E_{1}^{A S}$ and $E_{2}^{S}$ we obtain the absolute value of the phonon energy through Eq. (1).

lines whose separation is $20-100 \mathrm{~cm}^{-1}$ larger than $2 \hbar \omega_{\mathrm{LO}}$ or $20-100 \mathrm{~cm}^{-1}$ lower than $2 \hbar \omega_{\mathrm{TO}}$. In both cases we can register the Stokes and anti-Stokes spectra separated by $20-100 \mathrm{~cm}^{-1}$. As we see in Table I, there are several possibilities for each material. An important additional criterion is to select as $E_{1}^{L}$ the line with high scattering efficiency (to enhance the anti-Stokes peak). High scattering efficiency ${ }^{9}$ can be achieved by resonant effects (when the laser energy approaches the transition energy of some critical point in the interband spectra) and also by small absorption coefficient close to the direct gap. In practice, for a given material, all available laser lines can be checked for the Raman signal and those giving high scattering efficiency are good candidates for $E_{1}^{L}$. In Table I, we indicate the appropriate pairs of en- ergies by boldface. It is quite fortunate that some argon laser lines are close to resonances for $\mathrm{GaP}, \mathrm{InAs}, \mathrm{Ge}$, and $\mathrm{Si}$. For II-VI semiconductors, the optical-phonon energies are usually lower than in III-V so that $\hbar \omega_{0} / k T$ is around 1 at room temperature and the Stokes and anti-Stokes lines are of comparable intensity.

The simultaneous use of two laser lines is difficult to achieve because it would require multiline operation with special filters. However, it is much simpler to use the single line operation of the laser. If we use a charge coupled device (CCD) detector with a fixed grating position, we can take two subsequent spectra first using the $E_{1}^{L}$ line and then the $E_{2}^{L}$ line. In case we use a photomultiplier and we record the spectra with the grating moving step by step, we can change the laser line when the spectrometer scans the range between $E_{2}^{S}$ and $E_{1}^{A S}$. This was actually the method we used because the CCD detector usually offers poorer resolution than the photomultiplier.

In principle, it is possible to use higher power for the $E_{1}^{L}$ line than for the $E_{2}^{L}$ line to compensate for the lower intensity of the anti-Stokes signal. However, the higher power may cause broadening and shifting of the line. As long as one measures the changes of the Stokes/anti-Stokes separation with some external factor (strain, temperature), different powers of $E_{1}^{L}$ and $E_{2}^{L}$ are acceptable, provided they are kept constant during the experiment. We preferred to use the same power for $E_{1}^{L}$ and $E_{2}^{L}$ and to enhance the anti-Stokes signal by approaching some resonances with $E_{1}^{L}$.

In our experiments we used a XY-800 DILOR double spectrometer with a GaAs photomultiplier (Hamamatsu) and

TABLE I. Pairs of argon laser lines that can be used for the Stokes/anti-Stokes method in different semiconductors. The values in brackets indicate the separation of the considered lines in $\mathrm{cm}^{-1}$. The pairs for which the signal is enhanced (due to resonant effects) are in boldface. The LO and TO phonon energies for each material and an estimate of the Stokes/anti-Stokes intensity ratio for the LO phonon at room temperature ( $k T$ $=24 \mathrm{meV}$ ) are also listed.

\begin{tabular}{|c|c|c|c|c|}
\hline Semiconductor & $\begin{array}{l}\hbar \omega_{\mathrm{LO}} \\
\left(\mathrm{cm}^{-1}\right)\end{array}$ & $\begin{array}{l}\hbar \omega_{\mathrm{TO}} \\
\left(\mathrm{cm}^{-1}\right)\end{array}$ & $\exp \left(-\hbar \omega_{\mathrm{LO}} / k T\right)$ & $\begin{array}{l}\text { Pairs of argon laser lines (in } \mathrm{nm} \text { ) } \\
\text { and their separation }\left(\text { in } \mathrm{cm}^{-1} \text { ) in parens }\right.\end{array}$ \\
\hline $\mathrm{Si}$ & 520 & 520 & 15 & $\begin{array}{l}496-472(1014), 476-454(1016), 514-488 \\
(1056), 528-501(1018), 488-465 \\
(977), 501-476(1054), \mathbf{3 5 1}-\mathbf{3 6 3} \\
(994)\end{array}$ \\
\hline $\mathrm{Ge}$ & 300 & 300 & 4.8 & $\begin{array}{l}\mathbf{5 2 8 - 5 1 4}(522), \mathbf{5 1 4 - 4 9 6}(705), \\
\mathbf{5 1 4 - 5 0 1}(496), 501-488(560), 488-472 \\
(663), 472-457(684), 465-454 \\
(534)\end{array}$ \\
\hline GaAs & 289 & 266 & 4.5 & $\begin{array}{l}488-476(495), 514-501(496), 488-472 \\
(663), 472-457(684), 514-496 \\
(705), 476-465 \text { (482) }\end{array}$ \\
\hline InP & 343 & 306 & 6 & $\begin{array}{l}514-496 \text { (705), 501-488 (560), 528-514 } \\
(522), 465-454(534), 514-501 \\
(496), 488-476(495)\end{array}$ \\
\hline InAs & 238 & 217 & 3.5 & $\begin{array}{l}\mathbf{4 8 8}-\mathbf{4 7 6}(495), 496-488(351), 501-488 \\
(560), 465-454(534), 514-501 \\
(496), 528-514(522), 465-457(370)\end{array}$ \\
\hline $\mathrm{GaP}$ & 403 & 365 & 8.2 & $\begin{array}{l}\text { 514-496 (705), 496-476 (845), 476-457 } \\
(852), 472-454(847), 488-472 \\
(663), 472-457 \text { (684) }\end{array}$ \\
\hline
\end{tabular}




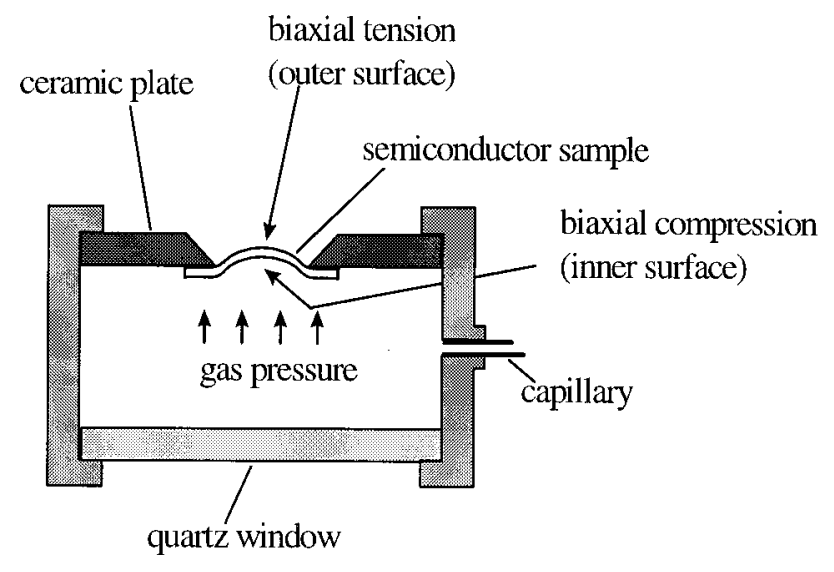

FIG. 2. Schematic cross section of the pressure cell used for the biaxial deformation studies. The semiconductor sample (thinned down to 20-70 $\mu \mathrm{m})$ is glued to a ceramic plate with a circular hole. This forms a diaphragm which is then deformed by gas pressure of a few bar (the gas is supplied from the reductor by a capillary). The inner surface of the diaphragm can be studied through the quartz window.

the photon counting technique. The monochromator step was 0.1 or $0.2 \mathrm{~cm}^{-1}$ and the count time of the detector ranged from 0.5 to $5 \mathrm{~s}$. The entrance and the exit slits were from 50 to $100 \mu \mathrm{m}$ and the middle slit was always twice that of the entrance slit. The excitation was by a LEXEL 95-3 argon laser. The power on the sample was typically $20-60 \mathrm{~mW}$ with the spot diameter between 50 and $100 \mu \mathrm{m}$. Raman peaks were fitted with standard curve-fitting procedures in order to achieve the required accuracy of Stokes/anti-Stokes separation. Phonon peaks were usually Lorentzian, whereas the plasma peaks had Gaussian shapes.

The application of biaxial strain was achieved by the diaphragm method described in Ref. 10. The sample was thinned (etched) down to $20-70 \mu \mathrm{m}$ and then glued to a ceramic plate with a circular hole $(2-5 \mathrm{~mm}$ in diameter). When the gas pressure is applied to the sample, we obtain biaxial strain in the center of the diaphragm, tensile on the outer surface, and compressive on the inner surface (see Fig. 2). Photoluminescence measurements on such diaphragms ${ }^{10}$ have shown that with a gas pressure of a few bar we obtain biaxial stresses of a few kbar. The deformation is roughly proportional to the square of the diameter-to-thickness ratio. We used pressures up to 20 bar supplied by a reductor on a He bottle. The importance of tunable biaxial strain in Raman measurements stems from the fact that Raman scattering has been used for the characterization of strained heteroepitaxial layers [like InGaAs on GaAs (Ref. 2) or GaAs on Si (Ref. 1)].

Theoretical expressions for the strain-induced shifts of phonon energies can be obtained from the model developed in Ref. 11 for the diamond structure. In that case, the triply degenerate $q=0$ optical phonon $\omega_{0}$ is split by biaxial strain into a singlet and a doublet. The shifts of the singlet $\Delta \omega_{s}$ and of the doublet $\Delta \omega_{d}$ are linear in strain and can be expressed by the phonon deformation potentials, $p, q$, and $r$, and the elastic constants $c_{11}, c_{12}$, and $c_{44}$. Biaxial experiments can be used to obtain $p, q$, and $r$ for different materials by using diaphragms with different crystallographic orientations. Our

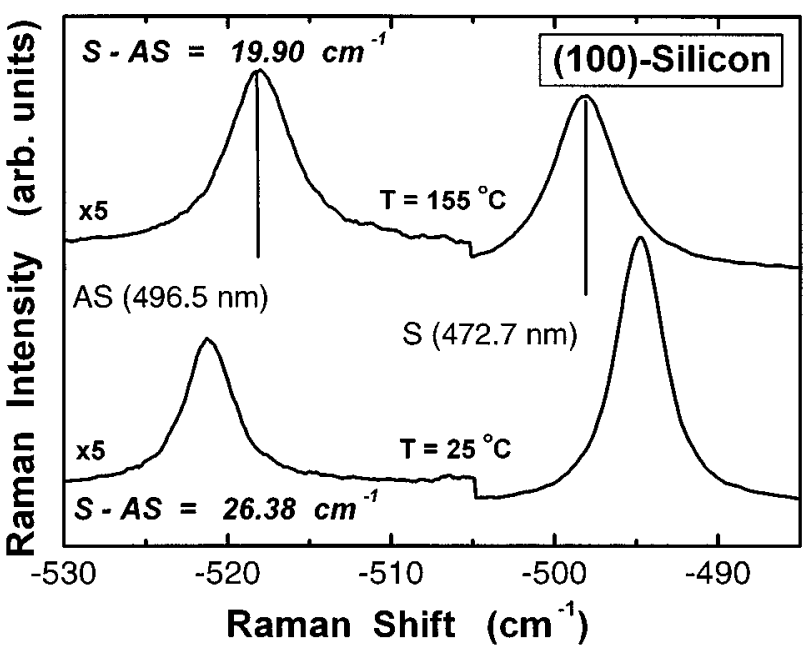

FIG. 3. Stokes/anti-Stokes spectra for $\mathrm{Si}(100)$ at two temperatures (excited by 496.5 and $472.7 \mathrm{~nm}$ lines). The anti-Stokes part was multiplied by 5 . The separation of Stokes and anti-Stokes peaks $(S-A S)$ is indicated for each spectrum.

Stokes/anti-Stokes method is useful in such studies as we will demonstrate in the following.

We also tested our method for studying the phonon shifts with laser power and with temperature. The temperature was varied between 300 and $600 \mathrm{~K}$ by gluing the samples with silver paste to electrically heated ceramic plates. The plates had a thermal expansion coefficient similar to that of the semiconductors which we studied in order to avoid the strain induced by temperature changes. The temperature of the plates was measured by a thermocouple placed close to the sample. With increasing temperature the anti-Stokes lines increased so our method works better.

A systematic review of the phonon shifts with biaxial strain and with temperature will be the subject of a separate paper. Here, we mainly want to demonstrate the usefulness of the Stokes/anti-Stokes method for five different materials that we studied.

\section{APPLICATION OF THE METHOD TO DIFFERENT SEMICONDUCTORS}

\section{A. Silicon}

For the strongest argon lines (514.5 and $488 \mathrm{~nm}$ ) the separation of the Stokes and anti-Stokes peaks is only about $15 \mathrm{~cm}^{-1}$, which is slightly too small while for $E_{1}^{L}$ $=496.5 \mathrm{~nm}$ and $E_{2}^{L}=472.7 \mathrm{~nm}$, the separation is around $26 \mathrm{~cm}^{-1}$ (see Table I). In Fig. 3 we show the spectra for these two lines at room temperature and at $155^{\circ} \mathrm{C}$ (with the anti-Stokes part multiplied by 5). The power on the sample (for both lines) was $30 \mathrm{~mW}$ and the zero of the monochromator was set at the 496.5 line (this is why the Raman shift shown is negative). Using accurate values for the laser energies from Ref. 12: $E_{1}^{L}=496.507 \mathrm{~nm}$ and $E_{2}^{L}=472.686 \mathrm{~nm}$, we obtain the room temperature value of the phonon energy in our sample to be equal to $520.68 \mathrm{~cm}^{-1}$. With increasing temperature, the separation between the Stokes and anti- 


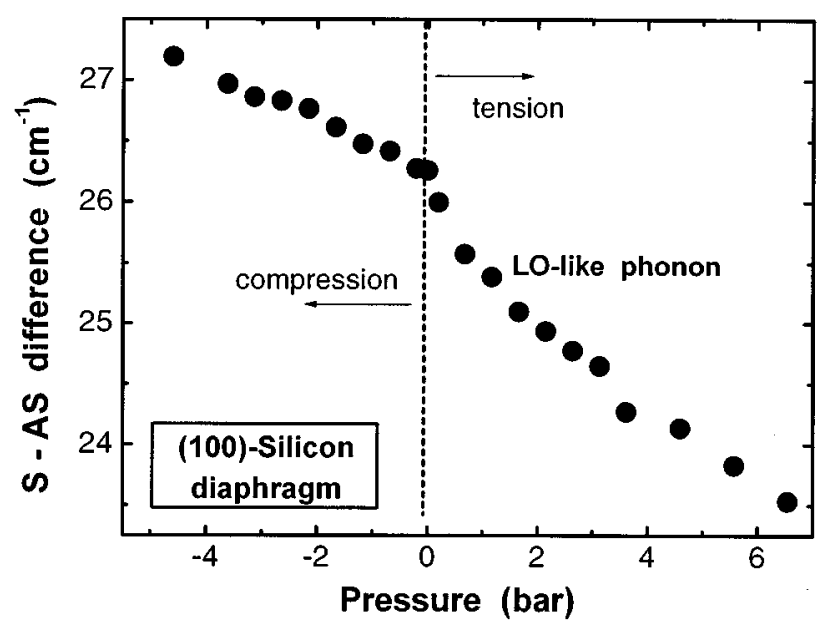

FIG. 4. Stokes/anti-Stokes separation for the LO phonon as a function of pressure applied to $\mathrm{Si}(100)$ diaphragm (1.7 $\mathrm{mm}$ radius, $70 \mu \mathrm{m}$ thickness). The spectra were taken in the center of the diaphragm on the outer (tension) and inner (compression) surface.

Stokes lines $(S-A S)$ decreases by around $6.48 \mathrm{~cm}^{-1}$, which means that the phonon energy is reduced by $3.24 \mathrm{~cm}^{-1}$. The linewidth increases substantially.

In Figs. 4 and 5 we present the results obtained on a 3.4 $\mathrm{mm}$ silicon diaphragm (70 $\mu \mathrm{m}$ thick) subject to pressure. In Fig. 4 the variation of the $S-A S$ separation (i.e., the double of the LO-phonon shift) in the middle of the diaphragm is shown for tensile and for compressive strain. The compression (tension) was obtained on the inner (outer) surface (see Fig. 2). Biaxial tension (compression) lowers (increases) the phonon energy. The effect is stronger for the tension than for the compression and is nonlinear with the applied pressure. In Fig. 5, we show the results of the radial scan of the inner surface of the diaphragm subject to a pressure of 3.6 bar. The compressive strain in the middle of the diaphragm becomes tensile at the edge and vanishes around $0.6 \mathrm{~mm}$ beyond the edge. The distribution of strain in silicon diaphragms is important for the design of piezoresistive pressure sensors ${ }^{13}$ and our method can be useful for such studies.

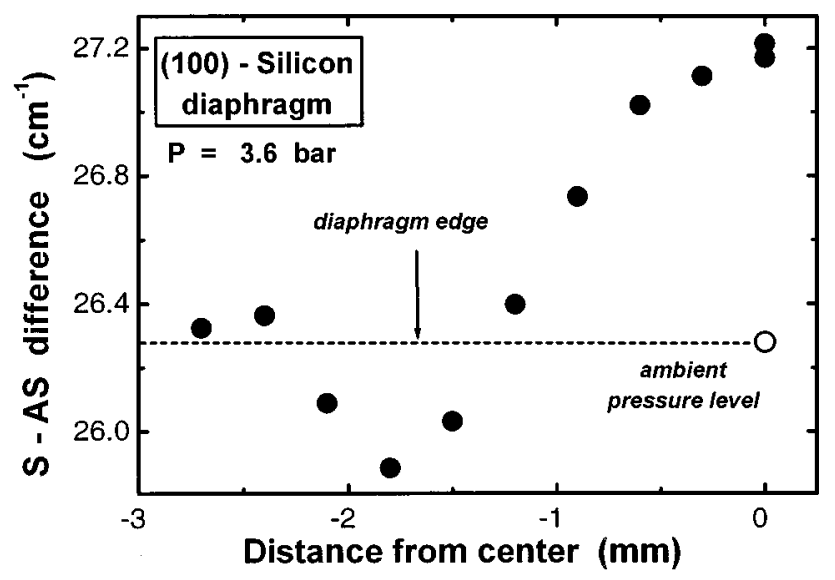

FIG. 5. Radial scan of the deformation (measured by the Stokes/anti-Stokes separation) on the inner surface of a $\mathrm{Si}(100)$ diaphragm subject to $3.6 \mathrm{bar}$ pressure $(1.7 \mathrm{~mm}$ radius, $70 \mu \mathrm{m}$ thickness).
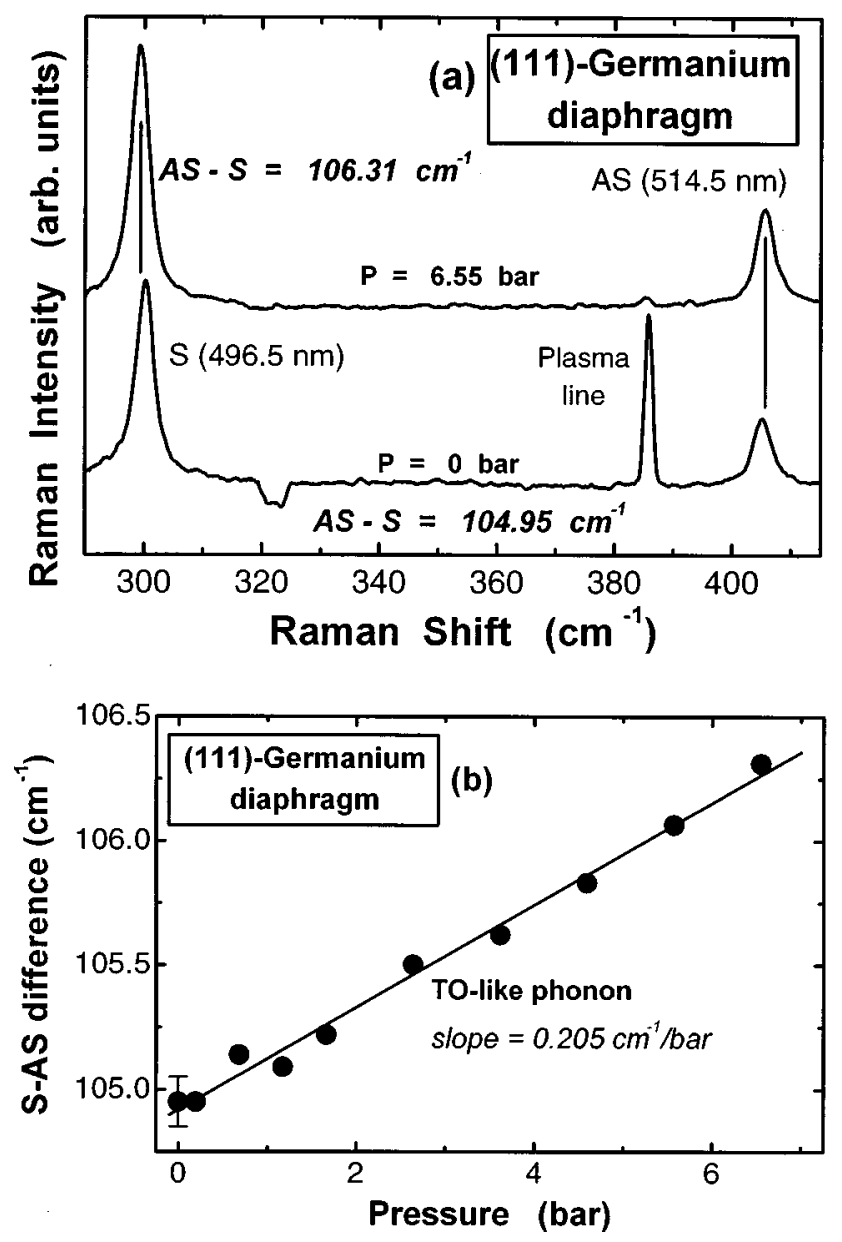

FIG. 6. Stokes/anti-Stokes separation (with 514.5 and $496.5 \mathrm{~nm}$ excitation) from the center of a $\mathrm{Ge}(111)$ diaphragm measured on the outer surface (tensile strain). The diaphragm was $3 \mathrm{~mm}$ in diameter and $60 \mu \mathrm{m}$ in thickness. (a) The spectra for $p=0$ and $p=6.55$ bar; (b) Stokes/anti-Stokes separation as a function of pressure.

\section{B. Germanium}

Here, the strongest signal is obtained for the 528.7 and $514.5 \mathrm{~nm}$ lines because of the proximity of the $E_{1}+\Delta_{1}$ resonance in interband transitions, ${ }^{14}$ which occurs at $540 \mathrm{~nm}$. Therefore, one of these lines can be selected for exciting the anti-Stokes peak. On our laser, the 528.7 line required a special mirror so we chose to work with the 514.5 and 496.5 lines. The power on the sample was $60 \mathrm{~mW}$ for each line. The zero of the monochromator was set at $496.5 \mathrm{~nm}$. For (111) samples, we used crossed polarizations (incident parallel to $(1 \overline{10})$ and scattered parallel to $(11 \overline{2})$ in order to see only one line (the doublet). In Fig. 6(a) we show the $S-A S$ spectra from the center of a $3 \mathrm{~mm}$ diaphragm (60 $\mu \mathrm{m}$ thickness). On some spectra, a narrow plasma line could be seen and it served as an additional reference. Plasma lines are very narrow (below $1 \mathrm{~cm}^{-1}$ ), so the spectrometer step has to be small to measure their position accurately. In Fig. 6(b) we summarize the results for tensile strain. The phonon shifts seem linear and they are around $-0.1 \mathrm{~cm}^{-1} / \mathrm{bar}$. Even though the effects are small $\left(1.4 \mathrm{~cm}^{-1}\right)$, the scatter of points is below $0.1 \mathrm{~cm}^{-1}$ so the overall accuracy is quite good. 

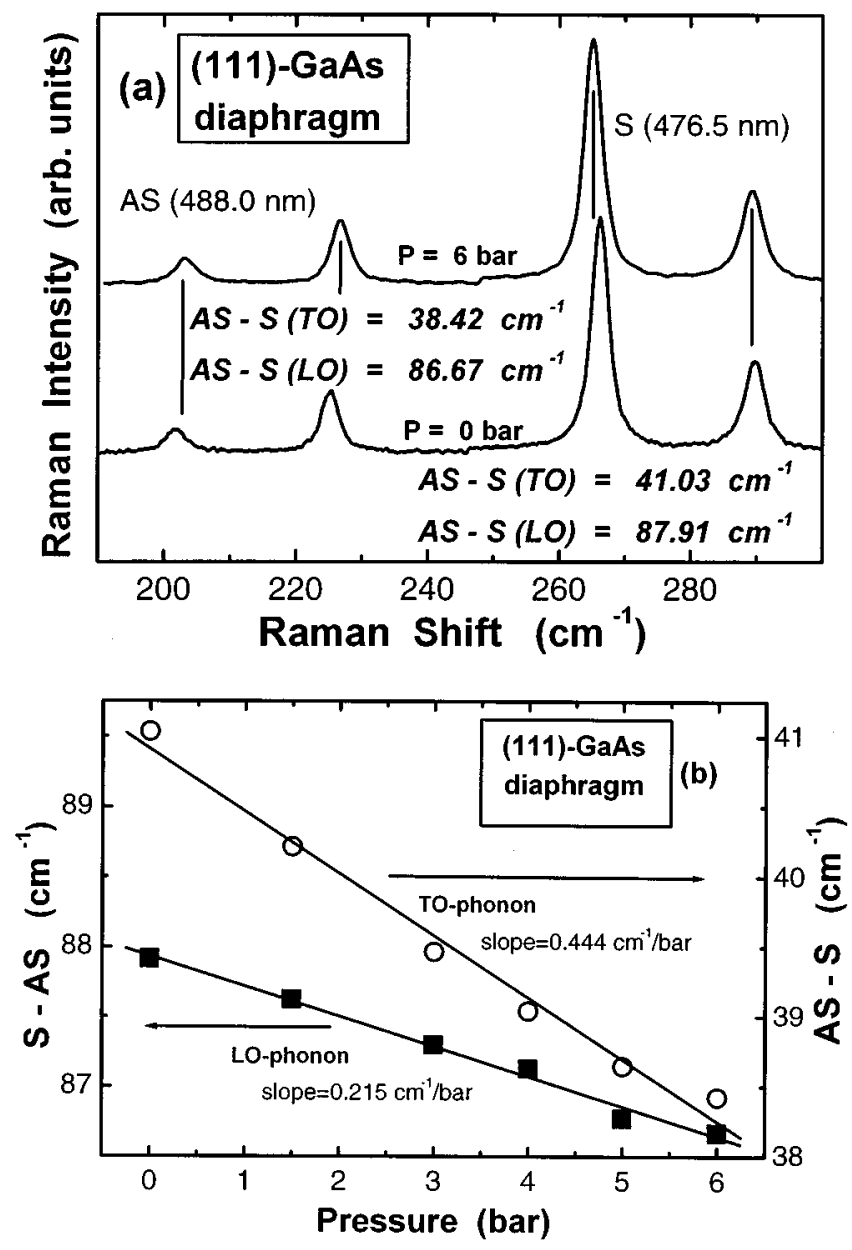

FIG. 7. (a) Stokes/anti-Stokes spectra (excited by 488 and $476.5 \mathrm{~nm}$ lines) from the center of a GaAs(111) diaphragm (3 mm diameter, $20 \mu \mathrm{m}$ thickness) for $p=0$ and $p=6$ bar (outer surface tension) and (b) Stokes/antiStokes separation for LO and TO phonons vs pressure.

The temperature change of the $S-A S$ separation for $\Delta T=130 \mathrm{~K}$ was $6 \mathrm{~cm}^{-1}$, slightly less than in $\mathrm{Si}$.

\section{Gallium arsenide}

For GaAs, different argon lines yield a similar Raman signal; the shortest wavelengths were only about $20 \%$ better than the longest. We chose the 488.0 and $476.5 \mathrm{~nm}$ argon lines which give a separation of the Stokes and anti-Stokes lines for the TO phonon close to $40 \mathrm{~cm}^{-1}$. The power on the sample was $60 \mathrm{~mW}$ for each line and zero of the monochromator was set at $476.5 \mathrm{~nm}$. For the (111) sample, the polarizations were both parallel to the $(1 \overline{10})$ direction in order to observe the TO and LO phonons. The spectra from the center of the GaAs(111) diaphragm (3 mm diameter, $20 \mu \mathrm{m}$ thickness) at zero and at 6 bar pressure are shown in Fig. 7(a). The variation of the $S-A S$ separation with pressure is shown in Fig. 7(b). With biaxial tension, the TO-phonon energy decreases twice as fast as the LO-phonon energy. This is in qualitative agreement with the predictions of the model of Ganesan et al. ${ }^{11}$ A quantitative comparison is difficult because of the large scatter of deformation potentials $p, q$, and $r$ reported in the literature. ${ }^{15}$

In Fig. 8 we show the evolution of the spectrum with

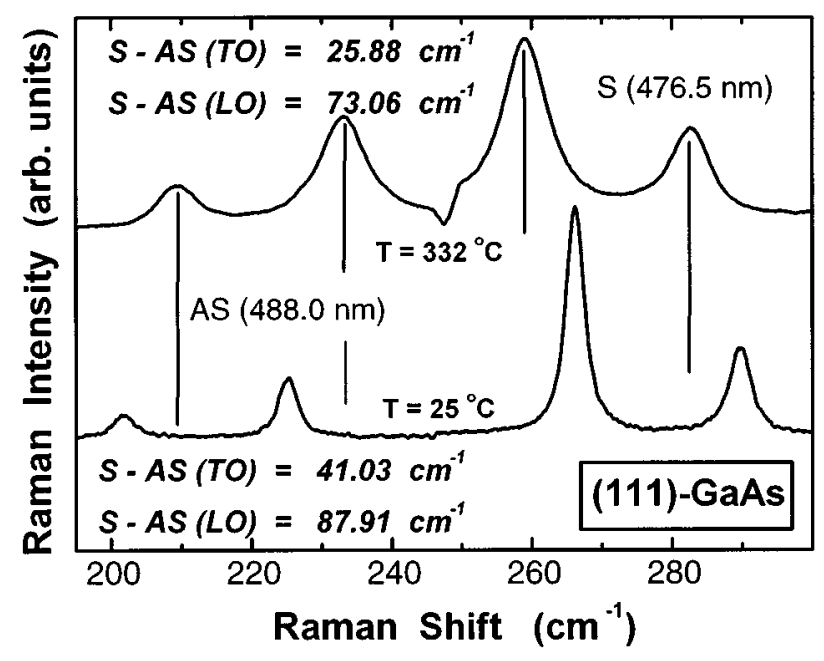

FIG. 8. Evolution of the Stokes/anti-Stokes spectra of (111) GaAs with temperature.

temperature. At $332{ }^{\circ} \mathrm{C}$ the anti-Stokes lines are of comparable intensity to the Stokes lines. The temperature shift is slightly larger for the TO than for the LO phonon. In Fig. 9 we present the $S-A S$ separation for the LO phonon in (100) GaAs as a function of the power incident on the sample (the laser spot was about $100 \mu \mathrm{m}$ in diameter). Figure 9 shows that there is a substantial dependence of the phonon energy on the laser power; no saturation can be observed down to 10 $\mathrm{mW}$. This is related to the strong absorption of the argon lines in GaAs so that the incident power is dissipated in a very narrow region close to the surface. That means that precise room temperature values of the phonon energies are hard to obtain and that in measurements of small shifts of these energies it is essential to keep the power on the sample constant.

\section{Indium arsenide}

The strongest Raman signal was obtained for the $488 \mathrm{~nm}$ line which is close to $E_{1}$ resonance for InAs. Therefore, we chose the same pair of lines as for GaAs: 488.0 and 476.5 $\mathrm{nm}$. The zero of the spectrometer was set at $476.5 \mathrm{~nm}$. In

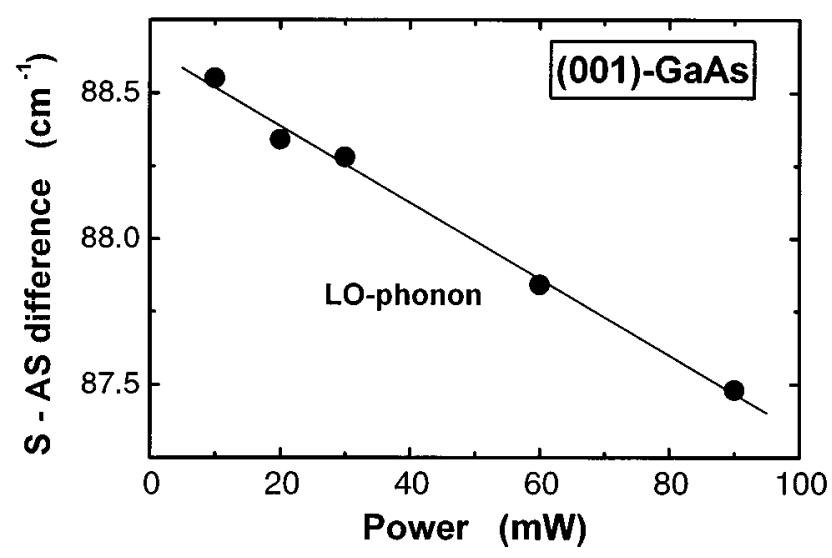

FIG. 9. Stokes/anti-Stokes separation for the LO phonon in $\operatorname{GaAs}(100)$ as a function of power incident on the sample (spot diameter $\sim 100 \mu \mathrm{m}$ ). 

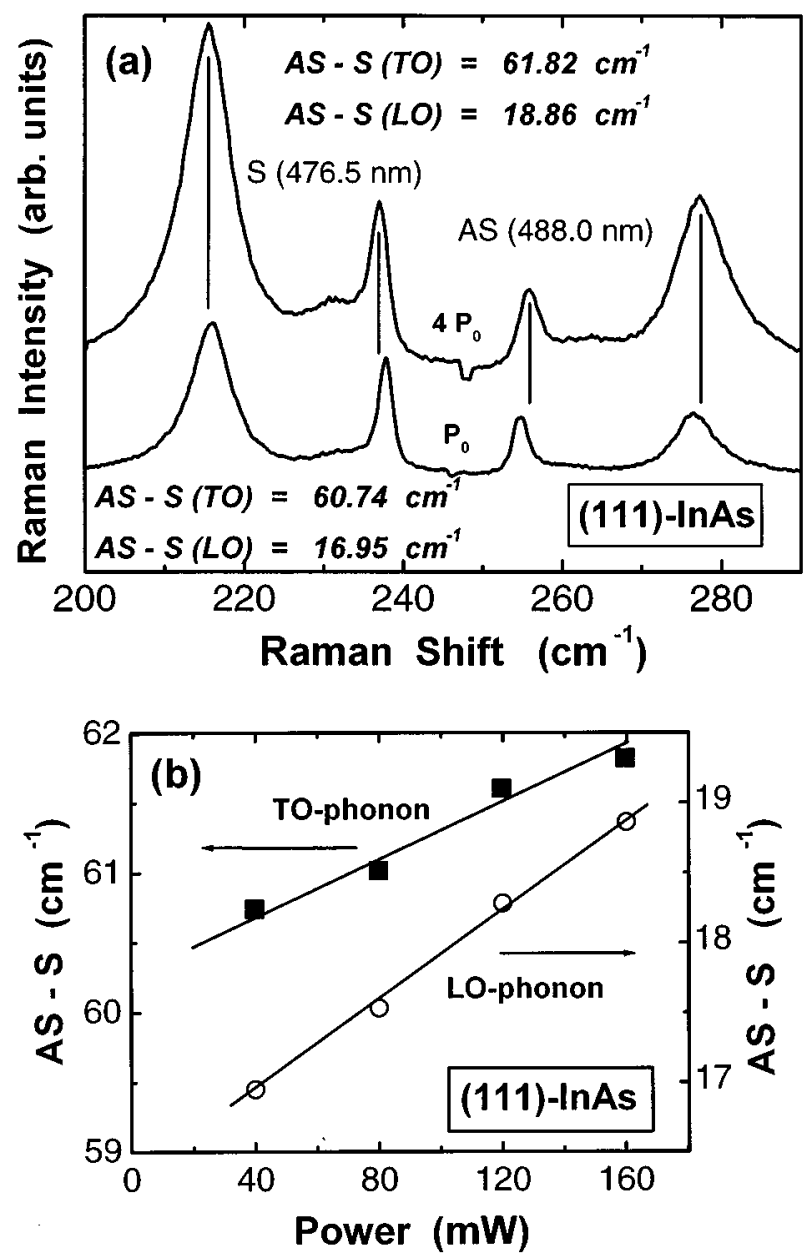

FIG. 10. (a) Stokes/anti-Stokes spectra (excited by 488 and $476.5 \mathrm{~nm}$ lines) from $\operatorname{InAs}(111)$ for two values of laser power $\left(P_{0}=40 \mathrm{~mW}\right)$ and (b) the Stokes/anti-Stokes separation for LO and TO phonons as a function of laser power.

Fig. 10(a) we show the spectra for two powers of the incident light [the polarizations were both parallel to $(1 \overline{1} 0)]$. The power dependence of the $S-A S$ separation [Fig. 10(b)]

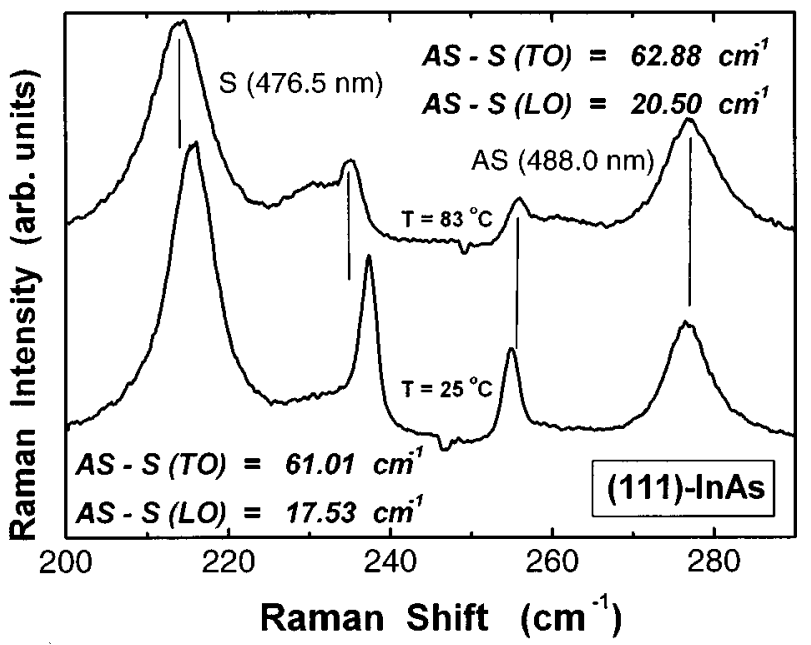

FIG. 11. Temperature variation of the Stokes/anti-Stokes spectrum in $\operatorname{InAs}(111)$.
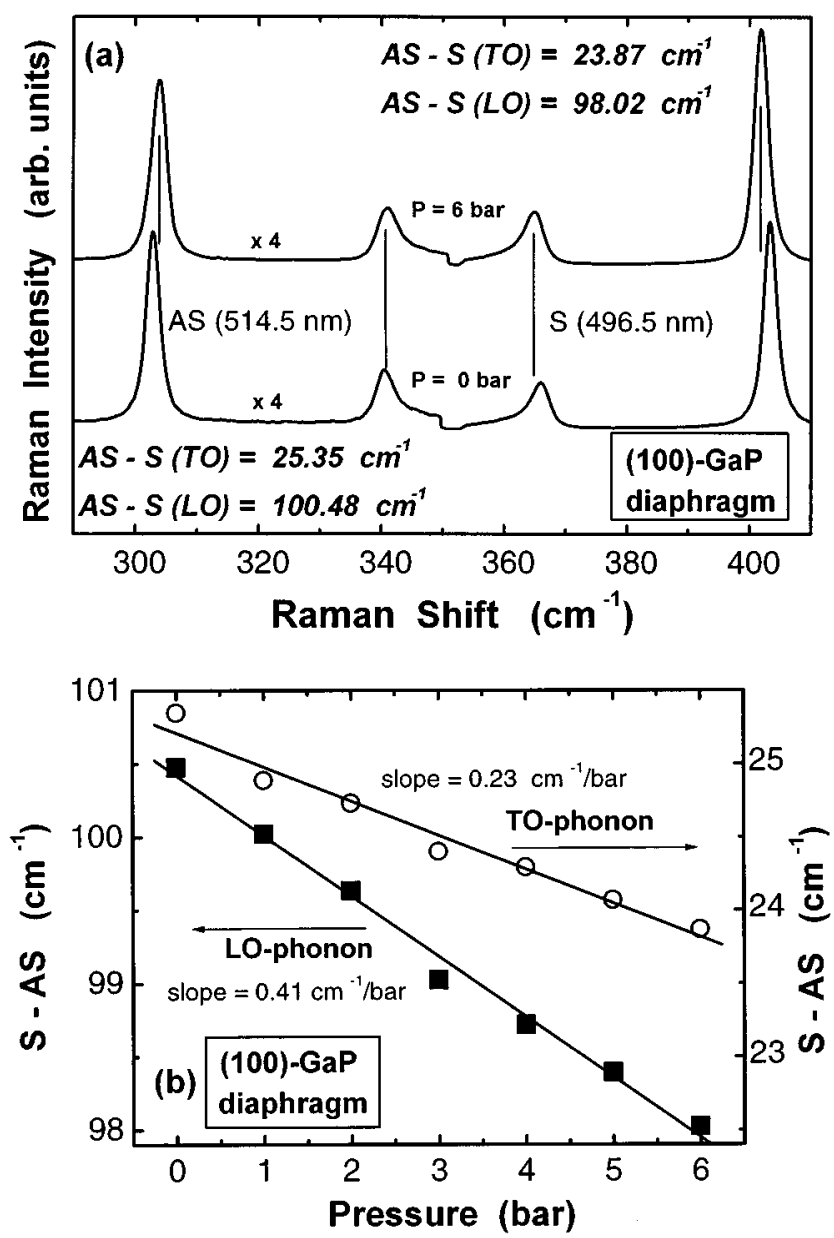

FIG. 12. (a) Stokes/anti-Stokes spectra (excited by 514.5 and $496.5 \mathrm{~nm}$ lines) from the center of $\mathrm{GaP}(100)$ diaphragm taken for Brewster-angle incidence for $p=0$ and $p=6$ bar (outer surface tension). The diaphragm was $3.4 \mathrm{~mm}$ in diameter and $30 \mu \mathrm{m}$ in thickness. (b) Stokes/anti-Stokes separation for LO and TO phonons vs pressure.

shows that there is no saturation in the range of powers applied and that the LO phonon shifts more than the TO. The same happens with increasing temperature (Fig. 11). At higher temperatures the LO phonon disappeared due to plasmon-phonon coupling; InAs is a narrow gap material and thermal excitation of carriers is very strong.

From the biaxial deformation experiments on diaphragms, we obtained a shift for the TO phonon $24 \%$ larger than for the LO phonon. This implies (using the theory of Ganesan et al. $)^{11}$ that the deformation potential $r / \omega_{0}^{2}$ should be much smaller than the value -0.76 obtained in Ref. 8 . More studies are necessary to elucidate this discrepancy.

\section{E. Gallium phosphide}

All visible argon lines are above the indirect gap in GaP ( $2.28 \mathrm{eV}$ at room temperature) but below the direct gap at $2.78 \mathrm{eV}$. Raman scattering is extremely intense because of the proximity of the resonance and because of the small absorption coefficient; Raman efficiency is proportional to the square of the scattering length. ${ }^{12}$ For this reason, the signal is strongest for the $514.5 \mathrm{~nm}$ line and we chose to work with 
514.5 and $496.5 \mathrm{~nm}$ lines (the same as for Ge). The zero of the monochromator was set at $496.5 \mathrm{~nm}$. The signal was so strong that the incident power could be set very low. However, this was not important because we observed no shift of the $S-A S$ separation (and no change of the linewidths) with increased laser power up to $100 \mathrm{~mW}$. The laser power is dissipated in a wide region of the sample (due to small absorption coefficient) and the heating effects are much smaller than in all the other materials that we studied.

In a (100) sample, we studied the effects of biaxial deformation using the Brewster-angle incidence. This geometry maximizes the electric-field component perpendicular to the (100) surface and allows for observation of both TO and LO phonons. The spectra from the center of the outer surface of the diaphragm (3.4 mm diameter, $30 \mu \mathrm{m}$ thickness) are shown in Fig. 12. For the biaxial strain in the (100) plane the shift of the LO phonon is stronger than that for the TO phonon [opposite to the (111) case]. This is in agreement with the theoretical predictions based on the model of Ref. 11 .

\section{SUMMARY AND CONCLUSIONS}

We have shown that the measurement of Stokes and anti-Stokes spectra excited by two lines of an argon laser yield absolute values of the optical phonon energies in semiconductors [see Eq. (1) and Fig. 1]. Small changes of these energies with strain, temperature, or with laser power can then be determined with high accuracy. The method avoids the need for reference lines and doubles the changes. We applied it to $\mathrm{Si}, \mathrm{Ge}, \mathrm{GaAs}$, InAs, and GaP. We showed that in several cases (Ge, InAs, and $\mathrm{GaP}$ ) the anti-Stokes signal can be enhanced by using resonant effects, i.e. by picking up appropriate pairs of lines for a given material (see Table I).

The method should be useful in micro-Raman studies of strain in silicon microstructures ${ }^{5,6}$ (see Fig. 5) for the determination of the phonon deformation potentials from uniaxial or biaxial experiments (Figs. 7 and 12) and for the studies of thermal shifts of the phonons (Figs. 3 and 8). Strain built into heteroepitaxial layers can be studied by Raman spectroscopy ${ }^{1-4}$ provided that the effects of alloying can be separated from those due to the strain. In these cases it is important that our method supplies the absolute phonon energies regardless of the calibration of the spectrometer.

The method fails at low temperature because of the vanishing of the anti-Stokes signal but the above-mentioned applications are usually performed at room temperature, where most devices operate.

\section{ACKNOWLEDGMENTS}

This work was supported by the Polish Committee for Research (KBN) through Grant No. 2P03B 04809. One of the authors (W.T.) gratefully acknowledges a fellowship by the Spanish Ministry of Education and Science and the hospitality of the staff at the Departamento de Física Aplicada, Universidad de Valencia.

${ }^{1}$ G. Landa, R. Carles, C. Fontaine, E. Bedel, and A. Munoz-Yague, J. Appl. Phys. 66, 196 (1989).

${ }^{2}$ M. Nakayama, K. Kubota, T. Kanata, H. Kato, S. Chika, and N. Sano, J. Appl. Phys. 58, 4342 (1985).

${ }^{3}$ M. K. Jackson, R. H. Miles, T. C. McGill, and J. P. Faurie, Appl. Phys. Lett. 55, 786 (1989).

${ }^{4}$ R. M. Abdelouhab, R. Braunstein, K. Barner, M. A. Rao, and H. Kroemer, J. Appl. Phys. 66, 787 (1989).

${ }^{5}$ I. De Wolf, Semicond. Sci. Technol. 11, 139 (1996).

${ }^{6}$ I. De Wolf, G. Pozzat, K. Pinardi, D. J. Howard, M. Ignat, S. C. Jain, and H. E. Maes, Microelectron. Reliab. 36, 1751 (1996).

${ }^{7}$ E. Anastassakis, A. Cantarero, and M. Cardona, Phys. Rev. B 41, 7529 (1990)

${ }^{8}$ F. Cerdeira, C. J. Buchenauer, F. H. Pollak, and M. Cardona, Phys. Rev. B 5, 580 (1972).

${ }^{9}$ P. Yu and M. Cardona, Fundamentals of Semiconductors (Springer, Berlin, 1995), p. 369.

${ }^{10}$ W. Trzeciakowski and T. P. Sosin, J. Phys. Chem. Solids 56, 649 (1994).

${ }^{11}$ S. Ganesan, A. A. Maradudin, and J. Oitmaa, Ann. Phys. 56, 556 (1970).

${ }^{12}$ A. R. Striganov and N. S. Sventitskii, Tables of Spectral Lines of Neutral and Ionized Atoms (Plenum, New York, 1968).

${ }^{13}$ Microsensors, edited by R. Miller, R. Howe, S. Senturia, R. Smith, and R. White (IEEE, New York, 1991).

${ }^{14}$ Numerical Data and Functional Relationships in Science and Technology, Landoldt-Börnstein New Series, Group III, Vol. 17a, edited by O. Madelung, M. Schulz, and H. Weiss (Springer, Berlin, 1982).

${ }^{15}$ S. Adachi, Physical Properties of III-V Semiconductor Compounds (Wiley, New York, 1992) 